\title{
Ground-state properties of polytypes of silicon carbide
}

\author{
P. J. H. Denteneer and W. van Haeringen \\ Department of Physics, University of Technology at Eindhoven, P. O. Box 513, \\ $N L-5600 \mathrm{MB}$ Eindhoven, The Netherlands
}

(Received 24 June 1985)

\begin{abstract}
The ground-state properties of cubic silicon carbide are calculated as a stepping stone towards a description of the polytypes of silicon carbide. The pseudopotential-density-functional method is used to calculate the self-consistent valence charge density as well as the equilibrium lattice constant and bulk modulus. Comparison with experimental data and earlier less rigorous calculations shows good agreement. A strategy is indicated to obtain a description of all polytypes.
\end{abstract}

\section{INTRODUCTION}

Polytypism is the occurrence of different modifications of one compound, consisting of identical layers of structure whose stacking sequence differs. ${ }^{1,2}$ The origin of polytypism is until now unexplained, although several theories exist, e.g., those based on screw dislocations ${ }^{3}$ or thermodynamic considerations. ${ }^{4}$

Polytypism in the silicon carbides $(\mathrm{SiC})$ - where it is studied most widely-is interesting for a number of other reasons. The polytypes of $\mathrm{SiC}$ are semiconductors with a varying band gap. They are natural superlattices and therefore pose problems similar to those found in calculations on artifically grown superstructures (e.g., large unit cells). The cubic modification $(3 C \mathrm{SiC})$ is the only IV-IV compound with zinc-blende structure and therefore is an intermediate between III-V semiconductors ( $\mathrm{GaAs}, \mathrm{GaP}$, etc.) and crystals with the diamond structure $(\mathrm{C}, \mathrm{Si}, \mathrm{Ge}, \alpha-\mathrm{Sn})$. They contain a first-row element in the sense of Phillips ${ }^{5}$ (carbon), usually leading to deviations from normal covalent behavior.

Although certain properties surely cannot be attained in this way (especially those depending strongly on temperature or details of the fabrication process), it is interesting to study the ground-state properties within the pseudopotential-density-functional scheme ${ }^{6}$ that has proved to be very successful already in calculating such properties for, e.g., $\mathrm{Si}$ and $\mathrm{C}^{7-9}$ The polytype that is easiest to handle in this scheme is the above-mentioned cubic modification $3 C$ (in the nomenclature of Ramsdell ${ }^{1,2}$ ) also named sphalerite or $\beta$-SiC. In Sec. II the method is outlined, together with a discussion of the procedure that is followed to reduce the computational work. Section III contains results of the calculations for $3 C$. At the end two concepts are presented that may be helpful in obtaining properties of other polytypes without resorting to the full-scale calculations of the scheme mentioned above.

\section{METHOD}

To obtain the self-consistent valence charge density $\rho$ we employ the pseudopotential-density-functional method ${ }^{6}$ using the norm-conserving pseudopotentials from Ref. 10 and plane-wave expansions. Two special $k$ points are used to integrate $\rho$ over the first Brillouin zone and the Wigner- interpolation formula is used to describe correlation effects. Further details and references are found in our earlier paper. ${ }^{11}$ From this self-consistently determined $\rho$ the total energy of the crystal can conveniently be calculated in momentum space. ${ }^{12}$

One-electron wave functions are expanded in plane waves (PW's) with kinetic energy less than $E_{\mathrm{PW}}$, a cutoff parameter to be varied to test convergence. It is considered the best strategy to keep $E_{\mathrm{PW}}$ fixed when varying lattice parameters or crystal structure. However, especially in the case of few special points this leads to non-negligible jumps in the total energy because discrete changes in the size of the basis set occur when the lattice parameters are varied. These jumps become negligible when the number of special points or the number of plane waves in the basis set (or, equivalently, $\left.E_{\mathrm{PW}}\right)$ is chosen sufficiently large. Since from a computing time point of view it is advantageous to choose both numbers as small as possible, we adopt the following strategy to reduce the effect of the jumps. (i) We use only two special points (for fcc lattices; an equivalent number has to be chosen for other types of lattices). (ii) We choose $E_{\mathrm{Pw}}$ so large that jumps in the total energy are about as large as they are for silicon in the diamond structure with two special points and $E_{\mathrm{PW}}=11.2 \mathrm{Ry}$. (For this value of $E_{\mathrm{PW}}$ a very good agreement with experiment was found for ground-state properties of $\mathrm{Si}$ in Ref. 8. However, the larger number of ten special points was used in that calculation.) (iii) Values of the lattice parameters for which the total energy is calculated are chosen not too close to each other nor to values for which discrete changes in the basis set occur. (For given $E_{\mathrm{PW}}$ and special points these values are known exactly.)

We have checked whether results obtained by this procedure are accurate enough by calculating for silicon (diamond structure, two special points; $E_{\mathrm{PW}}=11.2 \mathrm{Ry}$ ) the total energy at six lattice constants $a$ [ $a$ between 5.0 and $5.6 \AA$ and satisfying (iii)]. The results are fitted to the Murnaghan equation of state for solids (see Ref. 8). This results in an equilibrium lattice constant $a_{\text {eq }}$ of $5.447 \AA$ and a bulk modulus $B_{0}$ of $1.01 \mathrm{Mbar}$, values that are in excellent agreement with both earlier calculations ${ }^{8,13}$ and experimental values $\left[a_{\text {eq }}=5.43 \AA, B_{0}=0.99 \mathrm{Mbar}\right.$ (see Ref. 14)].

For $3 C$ (ii) implies that we have to choose $E_{\mathrm{PW}}$ as large as 29.7 Ry leading to about 380 PW's in the basis set if $a$ is near the experimental lattice constant of $4.36 \AA .^{14}$ In that case jumps are never larger than $2 \mathrm{mRy} /$ atom. 


\section{RESULTS}

In Fig. 1 we compare the valence charge density $\rho$ for $3 C$ obtained from a calculation with the empirical pseudopotential method ${ }^{15}$ (EPM) with one calculated self-consistently with norm-conserving pseudopotentials (SCNCP). In the EPM calculation the wave function was expanded in about 90 PW's, in the SCNCP calculation in about 380. Comparison of Figs. 1(a) and 1(b) shows that the empirical pseudopotential used for $\mathrm{Si}$ is too weak compared to the one used for $C$. This is less an effect of the locality of the atomic carbon pseudopotential-as was the case for the discrepancy between EPM- and SCNCP-charge densities in diamond ${ }^{11}$ - than of the fact that both potentials were not submitted to criteria ensuring transferability. For the band structure this appeared not to be such a drawback; ${ }^{15}$ for $\rho$ and therefore for ground-state properties it surely is. From Fig. 1(b) we see that $\rho$ displays features typical of ionic bonding (superposed spherical charge distributions centered on the atoms) as well as of covalent bonding (charge compressed in bonds between the atoms) illustrating that $3 C$ is partially ionic. For some semiconductors the position of the bond-charge maximum is a good measure of the degree of ionicity. ${ }^{5}$ For $3 C$, however, this is not true; the bond-charge maximum is positioned 0.79 a.u. from the carbon atomic position and this would make SiC more ionic than GaAs, which it definitely is not (the ionicity according to Ref. 5 is 0.18 for $\mathrm{SiC}$ and 0.31 for $\mathrm{GaAs}$ ). Therefore $\mathrm{SiC}$ is an example of the empirical rule ${ }^{5}$ that compounding a first-row element (supplemented by $\mathrm{Na}, \mathrm{Mg}$, and $\mathrm{Al}$ ) with other elements leads to deviations from normal covalent behavior. An earlier calculation based on a combination of the Gordon-Kim theory and the Weber bond-charge model ${ }^{16}$ resulted in a position of the bond charge 1.26 a.u. from the carbon atom.
In Table I the results of total energy $\left(E_{\text {total }}\right)$ calculations for $3 C$ are summarized. $E_{\text {total }}$ was evaluated for eight values of the lattice constant with $E_{\mathrm{PW}}=29.7 \mathrm{Ry}$ (see Sec. II); each time the self-consistency process was stopped when $E_{\text {total }}$ was stable to within $10^{-4} \mathrm{Ry} /$ cell. The results were fitted to the equation of state for solids by Murnaghan which contains $a_{\text {eq }}, B_{0}$, the pressure derivative of the bulk modulus $B_{0}^{\prime}$, and the minimum total energy $E_{\text {tot, min }}$ as parameters. ${ }^{17}$ The rms error of this fit was $10^{-3} \mathrm{Ry} /$ cell and the results are compared in Table I with similar calculations using $E_{\mathrm{PW}}=20.6 \mathrm{Ry}$ and with experimental values.

The experimental value of $E_{\text {tot, min }}$ is obtained as follows. An estimated value for the energy of the crystal $E_{\text {crystal }}$ expressed in experimentally accessible quantities is given by a slight generalization of formula (12) in Ref. 18:

$$
E_{\text {crystal }}=-\left(\sum_{i=1}^{4} \phi_{i}(\mathrm{C})+\sum_{i=1}^{4} \phi_{i}(\mathrm{Si})+E_{\text {coh }}(3 C)\right),
$$

where the $\phi_{i}$ are ionization potentials of the atom ${ }^{14}$ and $E_{\text {coh }}$ $(3 C)$ is the cohesive energy of $3 C,\left[E_{\text {coh }}(3 C)=0.931\right.$ $\mathrm{Ry} /$ cell] following from the cohesive energies for $\mathrm{Si}$ and $\mathrm{C}$ and the heat of formation of $3 C .{ }^{14}$ To get the equivalent to the total energy as given by our calculations we have to subtract the energy $E_{\text {vib }}$ due to zero-temperature vibrations of the atoms. This last energy, which is small but not negligible, may be estimated to be $E_{\text {vib }}=\frac{9}{8} k_{B} \Theta_{D}$ (per atom ${ }^{19}$ ), where $k_{B}$ is Boltzmann's constant and $\Theta_{D}$ the Debye temperature. Since $\Theta_{D}=1430 \mathrm{~K}$ for $3 C$ at $T=0 \mathrm{~K}$ (Ref. 14) we have $E_{\text {vib }}=0.020 \mathrm{Ry} /$ cell.

From Table I we see that for $E_{\mathrm{PW}}=29.7 \mathrm{Ry}, E_{\mathrm{tot} \text {, } \min }$ is still not fully converged. We remark that our value is closer to the experimental estimate for $E_{\text {tot,min }}$ than the result that
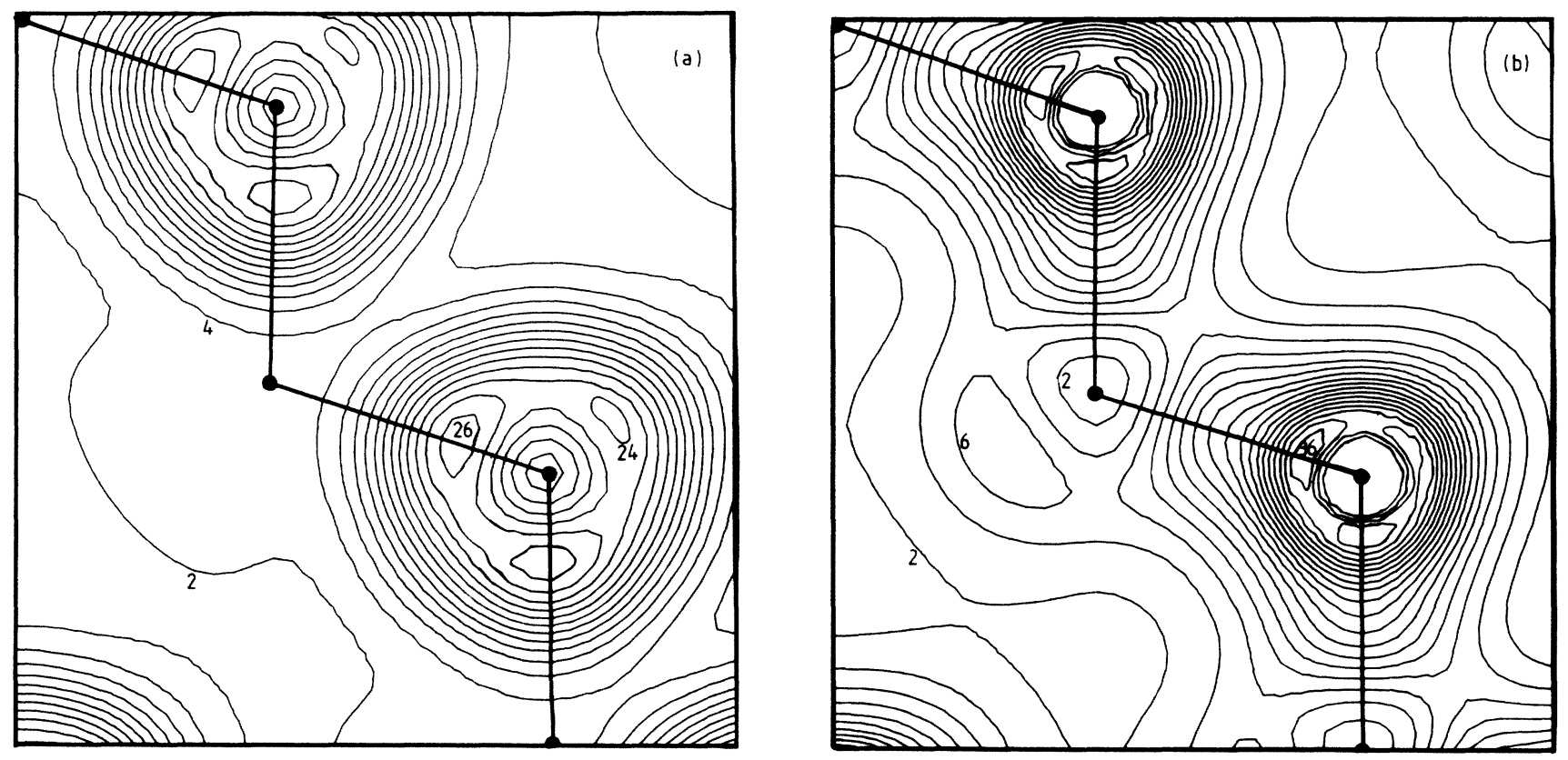

FIG. 1. Total valence charge density for $3 C \mathrm{SiC}$ at the experimental lattice constant in the (110) plane. Black dots indicate atomic positions (carbon atom at the higher-density position) and thick lines connect nearest neighbors. Units are electrons per unit cell volume with a contour step of two. (a) Calculated by the empirical pseudopotential method. (b) Calculated by the self-consistent pseudopotential method using norm-conserving pseudopotentials. Some contours near the carbon atom have been omitted for clarity. 
TABLE I. Equilibrium lattice constant $a_{\mathrm{eq}}$, bulk modulus $B_{0}$, pressure derivative of bulk modulus $B_{0}^{\prime}$, and minimum total energy $E_{\text {tot,min }}$ for $3 C \mathrm{SiC}$ (definition of $E_{\mathrm{PW}}$ in the text).

\begin{tabular}{lccc}
\hline & $E_{\mathrm{PW}}=20.6 \mathrm{Ry}$ & $E_{\mathrm{PW}}=29.7 \mathrm{Ry}$ & Experiment $^{\mathrm{a}}$ \\
\hline$a_{\mathrm{eq}}(\AA)$ & 4.411 & 4.365 & 4.360 \\
$B_{0}(\mathrm{Mbar})$ & 3.08 & 2.00 & $\cdots$ \\
$B_{0}^{\prime}$ & 2.1 & 7.3 & $\cdots$ \\
$E_{\mathrm{tot} \text { min }}(\mathrm{Ry} / \mathrm{cell})$ & -19.140 & -19.309 & -19.410 \\
\hline
\end{tabular}

a Reference 14.

may be deduced from Ref. 16, which is $-19.258 \mathrm{Ry} /$ cell. (The dissociation energy $D_{e}$ in Ref. 16 is equivalent to minus our $E_{\text {crystal. }}$ ) Concerning the convergence with respect to an increase of $E_{\mathrm{PW}}$ of the other quantities in Table I we recall that $E_{\mathrm{PW}}$ is chosen so large $(29.7 \mathrm{Ry})$ that the uncertainty in energy differences is the same as for silicon with $E_{\mathrm{PW}}=11.2 \mathrm{Ry}$ (see Sec. II). Since energy differences are all that are needed to determine $a_{\mathrm{eq}}, B_{0}$, and $B_{0}^{\prime}$, we expect that these quantities have converged to the same degree as in Ref. 8: better than $1 \%$ for $a_{\text {eq }}$ and about $5 \%$ for $B_{0}$ and $B_{0}^{\prime}$. The results for $E_{\mathrm{PW}}=20.6 \mathrm{Ry}$ only illustrate that $a_{\text {eq }}$ converges faster than $B_{0}$, which in turn converges faster than $B_{0}^{\prime}$.

Another type of uncertainty in the results of Table $I$ is due to the fitting. From fits to different forms of the equation of state and to subsets of our results for $E_{\text {total }}$ we estimate this uncertainty to be $7.10^{-4} \mathrm{Ry} /$ cell for $E_{\mathrm{tot} \text { min }}$, better than $0.3 \%$ for $a_{\text {eq }}$, about $10 \%$ for $B_{0}$, whereas $B_{0}^{\prime}$ can vary by a factor of 2 .

Our calculated $a_{\mathrm{eq}}$ is in excellent agreement with experiment, while $B_{0}$ and $B_{0}^{\prime}$ are beyond direct comparison because of lack of experimental results. The latter quantities are difficult to measure because the relatively large crystals that are needed are hard to fabricate. There are two facts in support of our value of $B_{0}$ : (i) it is intermediate between bulk moduli of $\mathrm{Si}$ and $\mathrm{C}(0.99$ and $4.42 \mathrm{Mbar}$, respectively) and (ii) it agrees with a theoretical calculation by Tolpygo ${ }^{20}$ of the elastic constants $c_{11}$ and $c_{12}$. These are related to $B_{0}$ by the equality $B_{0}=\left(c_{11}+2 c_{12}\right) / 3$ (cubic crystals ${ }^{21}$ ). Using this equality, Ref. 20 gives $B_{0}=2.11 \mathrm{Mbar}$. An unexplained fact is the value of $B_{0}$ in the literature ${ }^{14}$ for a hexagonal modification of $\mathrm{SiC}$ (which one exactly is not stated). This value of $0.97 \mathrm{Mbar}$ differs by more than a factor of 2 from our $B_{0}$, which is somewhat surprising for two crystals constituted of the same atoms and with like structures.

We end by indicating how these results for $3 C$ may be used to come to a description of ground-state properties of all polytypes. As polytypes differ only in the way of stacking identically structured layers, it is tempting to assume that knowledge of the electron density in one layer of one polytype (e.g., $3 C$ ) is enough to construct the electron density in all layers of all polytypes. If, furthermore, the functional dependence of total energy on density were known, we then would have access to the total energy of all polytypes. Our assumption is not contradicted by a calculation of the self-consistent valence charge density $\rho$ in the polytype with the wurtzite structure $(2 \mathrm{H} \mathrm{SiC}$, four atoms in the unit cell). In Fig. 2 this $\rho$ is compared with one calculated
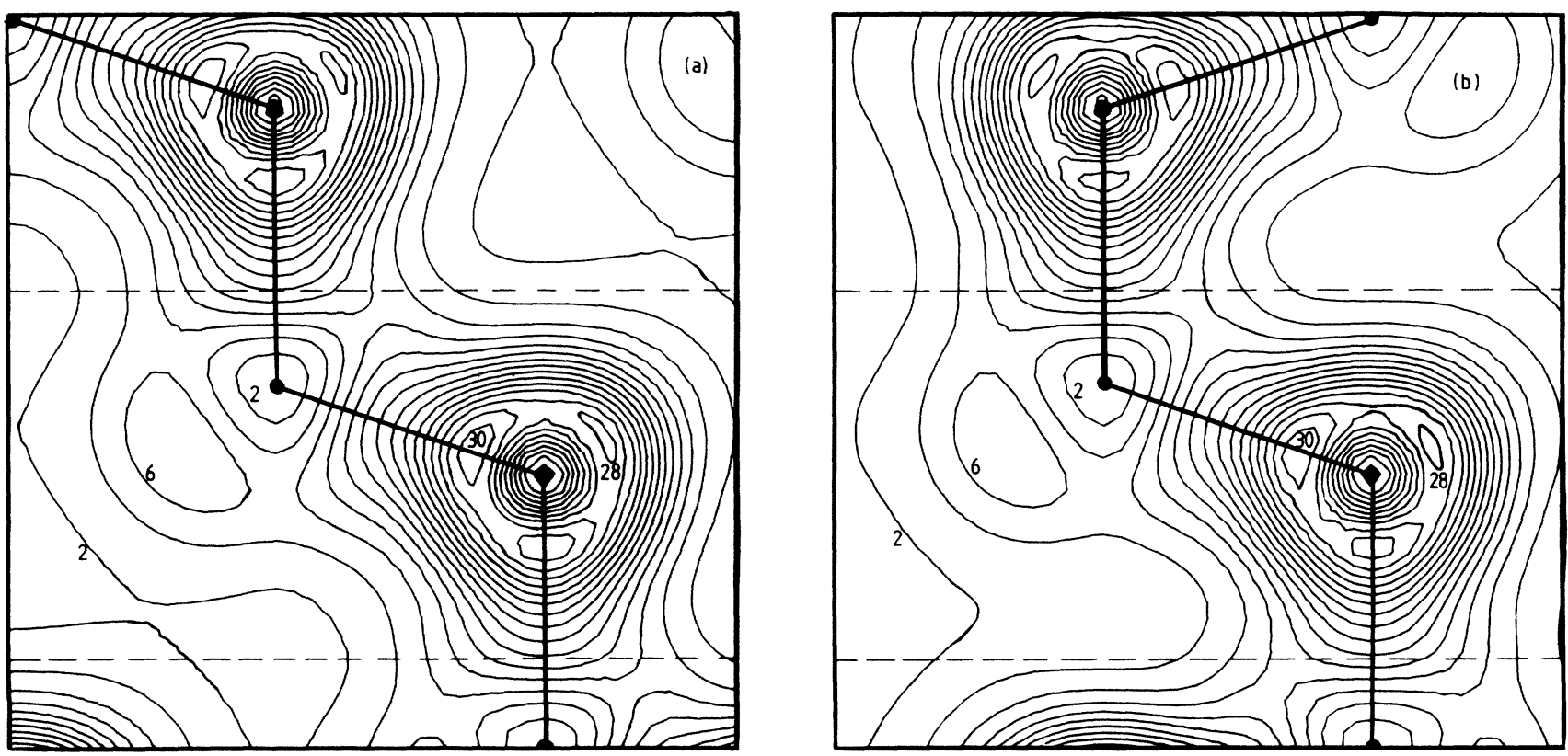

FIG. 2. Total valence charge density for $3 C$ and $2 H$ in equivalent planes. Units are electrons per unit cell volume of $3 C$ with a contour step of two. (Marks as in Fig. 1). The central layer is the layer between dashed lines (see text). (a) $3 C$ and (b) $2 H$. 
for $3 C$ in the same plane [the $c$ axis of $2 H$ is equivalent to the (111) direction in $3 C$ ] for the same value of $E_{\mathrm{PW}}$ $(=15.2 \mathrm{Ry})$. The agreement in the central layer seems rather good, while densities in the top and bottom layer displayed in Fig. 2 may be mapped upon each other by rotation over $\pi$ around the bond axes (keeping the central layer fixed).

Another aspect of polytypes that may be helpful in deducing properties of the polytypes from elaborate calculations (like the ones described above) for a few (simple) polytypes only is that of interpolation. When polytypes are described in terms of the well-known hexagonality parameter $h$ only, the cubic $(3 C)$ and wurtzite $(2 H)$ modifications are extremes in the hexagonality scale with $h=0$ and 1 , respectively. An analysis of the Ewald constants $\alpha_{\text {Ewald }}(h)$-i.e., the structure-dependent constants related to the ion-ioninteraction energy ${ }^{8,12}$ - for the respective polytypes ${ }^{22}$ has shown that within an accuracy of 1 in $10^{5}$ the Ewald constants may be obtained for any polytype from knowledge of $\alpha_{\text {Ewald }}$ for $3 C$ and $2 H$ only.

At the moment work is in progress to investigate whether these two concepts of "charge-density mapping" and "interpolation" are indeed powerful enough to obtain groundstate properties of polytypes of silicon carbide.
${ }^{1}$ A. R. Verma and P. Krishna, Polymorphism and Polytypism in Crystals (Wiley, New York, 1966).

${ }^{2}$ W. F. Knippenberg, Philips Res. Rep. 18, 161 (1963).

${ }^{3}$ F. C. Frank, Philos. Mag. 42, 104 (1951).

${ }^{4} \mathrm{H}$. Jagodzinski, Neues Jahrb. Mineral. Monatsh. 3, 49 (1954).

5 J. C. Phillips, Bonds and Bands in Semiconductors (Academic, New York, 1973)

${ }^{6}$ M. Schlüter, J. R. Chelikowsky, S. G. Louie, and M. L. Cohen, Phys. Rev. B 12, 4200 (1975).

${ }^{7}$ M. T. Yin and M. L. Cohen, Phys. Rev. B 24, 6121 (1981).

${ }^{8}$ M. T. Yin and M. L. Cohen, Phys. Rev. B 26, 5668 (1982).

9J. R. Chelikowsky and S. G. Louie, Phys. Rev. B 29, 3470 (1984).

${ }^{10}$ G. B. Bachelet, H. S. Greenside, G. A. Baraff, and M. Schlüter, Phys. Rev. B 24, 4745 (1981).

${ }^{11}$ P. J. H. Denteneer and W. van Haeringen, J. Phys. C 18, 4127 (1985).

12 J. Ihm, A. Zunger, and M. L. Cohen, J. Phys. C 12, 4409 (1979).

${ }^{13}$ O. H. Nielsen and R. M. Martin, Phys. Rev. Lett. 50, 697 (1983).

${ }^{14}$ Landolt-Börnstein: Numerical Data and Functional Relationships in Science and Technology, edited by $\mathrm{O}$. Madelung (Springer-Verlag, Berlin, 1982), Group 3, Vol. 17, Part a; C. E. Moore, Ionization
Potentials and Ionization Limits Derived from the Analysis of Optical Spectra, U.S. National Bureau of Standards, Ref. Data Ser. 34, (U.S. GPO, Washington, DC, 1970); Handbook of Chemistry and Physics, 63rd ed., edited by R. C. Weast (CRC, Boca Raton, FL, 1982).

${ }^{15} \mathrm{H}$. G. Junginger and W. van Haeringen, Phys. Status Solidi (b) 37, 709 (1970).

${ }^{16}$ C. Muhlhausen and R. G. Gordon, Phys. Rev. B 24, 2161 (1981).

${ }^{17}$ The results for $B_{0}$ and $B_{0}^{\prime}$ were obtained by fitting of the subset of six points lying closest to $a_{\text {eq }}$. Since $B_{0}$ and $B_{0}^{\prime}$ are related to the second and third derivative of $E_{\text {total }}$ as a function of volume, respectively, in this way the effect of numerical uncertainties is reduced.

18 J. Ihm and M. L. Cohen, Phys. Rev. B 21, 1527 (1980).

${ }^{19} \mathrm{M}$. Born and K. Huang, Dynamical Theory of Crystal Lattices (Oxford, London, 1954).

${ }^{20} \mathrm{~K}$. B. Tolpygo, Fiz. Tverd. Tela (Leningrad) 2, 2655 (1960) [Sov. Phys. Solid State 2, 2367 (1961)].

21 J. F. Nye, Physical Properties of Crystals (Oxford, London, 1957).

${ }^{22}$ D. Lenstra, A. G. Roosenbrand, P. J. H. Denteneer, and W. van Haeringen, Physica B (to be published). 